\title{
Interaction Paradigms Versus Age-Related User Profiles: an Evaluation on Content Selection
}

\author{
D. C. M. Carvalho, M. E. C. Bessa, L. G. M. Magalhães, and E. M. E. M. Carrapatoso
}

\begin{abstract}
Novel input modalities such as touch, tangibles or gestures try to exploit human's innate skills rather than imposing new learning processes. However, despite the recent boom of different natural interaction paradigms, it hasn't been systematically evaluated how these interfaces influence a user's performance or whether each interface could be more or less appropriate when it comes to: 1) different age groups; and 2) different basic operations, as data selection, insertion or manipulation. This work presents the first step of an exploratory evaluation about whether or not the users' performance is indeed influenced by the different interfaces. The key point is to understand how different interaction paradigms affect specific target-audiences (children, adults and older adults) when dealing with a selection task. 60 participants took part in this study to assess how different interfaces may influence the interaction of specific groups of users with regard to their age. Four input modalities were used to perform a selection task and the methodology was based on usability testing (speed, accuracy and user preference). The study suggests a statistically significant difference between mean selection times for each group of users, and also raises new issues regarding the "old" mouse input versus the "new" input modalities.
\end{abstract}

Keywords- Human-Computer Interaction, Natural User Interfaces, Interaction Paradigms, Input Modalities, Mouse, Touch, Tangibles, Gestures, Selection Task.

\section{INTRODUÇÃO}

$\mathrm{O}$ PARADIGMA de interação pessoa-computador tem vindo a sofrer alterações ao longo das últimas décadas. $\mathrm{O}$ modo tradicional de input / output baseado em interfaces WIMP (Windows, Icons, Menus, Pointing device) tem vindo a evoluir de encontro a uma interação orientada ao utilizador e ao tipo de tarefa a desempenhar [1]. Efetivamente, cada vez mais é dada maior importância às necessidades do utilizador e às suas capacidades inatas, numa tentativa de inclusão digital de toda a população [2].

Esta gradual alteração de paradigma para interfaces consideradas mais naturais, ditas pós-WIMP [3], admite ao utilizador tirar partido das suas aptidões inatas e evita a necessidade de novos processos de aprendizagem. Efetivamente, interfaces baseadas na interpretação de comportamentos humanos complexos, como é o caso da fala, movimentos oculares, toque, gestos e linguagem corporal,

D. C. M. Carvalho, INESC TEC, Porto, e Universidade de Trás-osMontes e Alto Douro (UTAD), Vila Real, Portugal, dianac@utad.pt

M. E. C. Bessa, INESC TEC, Porto, e Universidade de Trás-os-Montes e Alto Douro (UTAD), Vila Real, Portugal, maxbessa@utad.pt

L. G. M. Magalhães, INESC TEC, Porto, e Universidade de Trás-osMontes e Alto Douro (UTAD), Vila Real, Portugal, lmagalha@utad.pt

E. M. E. M. Carrapatoso, Faculdade de Engenharia da Universidade do Porto (FEUP), Porto, Portugal, emc@fe.up.pt requerem uma carga cognitiva mais baixa e permitem-nos manipular conteúdos digitais mais intuitivamente [4], [5].

Considera-se um modo de interação quando existe uma combinação de determinado tipo de input e respetivo output [6], podendo ser reconhecida de forma independente, ou numa mesma interface multimodal. Esta última pode reconhecer dois ou mais modos de interação, tornando-se mais flexível na forma como apreende os comandos do utilizador e que tipos de input permite de acordo com as suas aptidões e preferências, nomeadamente idade, língua nativa, níveis de literacia digital, estilos cognitivos, deficiências motoras, ou mesmo doenças temporárias [7]. Da mesma forma, de acordo com [8], estas interfaces estão igualmente cientes do contexto espacial e geográfico do utilizador, tornando-se assim mais robustas e eficientes quando lidam com informação visual e espacial. Recentes interfaces multimodais reconhecem até dois modos de interação em diferentes áreas de interesse. Seja em combinações de pens e fala [9], [10], fala e gestos [11], [12], multitoque e objetos tangíveis [13], [14].

Contudo, com a constante adição de novos modos de input no nosso dia-a-dia, ainda não existe uma consciência real sobre quais os paradigmas que podem ajudar na interação ou, pelo contrário, prejudicar a performance do utilizador. Dessa forma, seria importante perceber realmente quais os que se consideram como facilitadores na interação mediante determinados contextos ou perfis de utilização (como crianças, pessoas com diferentes níveis de literacia digital, idosos, etc). Mais ainda, também as tarefas a desempenhar por parte do utilizador poderão ser influenciadas pela interface escolhida. Realmente, cada tarefa pode ser influenciada pelo sentido de naturalidade, eficiência e graus de liberdade (DOF - Degrees of Freedom) proporcionados ao utilizador [15]. Na execução das tarefas mais básicas de um ambiente virtual (seleção, inserção e manipulação), é crucial compreender se existem modos de interação mais eficientes do que outros de forma a auxiliar na interação, ou seja, qual a/as interfaces mais adequadas para diferentes tarefas.

Este artigo justifica-se, uma vez que não temos conhecimento que tenha sido apresentado na literatura um estudo sistemático para perceber a relação entre as interfaces versus diferentes tarefas e perfis de utilizador, de forma a perceber que modos de interação são considerados melhores ou piores mediante tarefas específicas.

Neste contexto, este estudo comporta a primeira fase de uma abordagem mais exaustiva que tem como objetivo final compreender até que ponto a redundância de comandos e constante adição de novos paradigmas de interação começam a saturar e prejudicar o desempenho do utilizador. Entretanto, como ponto de partida para tal observação torna-se necessário 
entender a relação do utilizador com diferentes interfaces individuais (que apenas permitem um modo de interação), bem como se o tipo de tarefa e/ou perfil de utilizador influencia esta mesma relação. Assim sendo, neste artigo almejamos perceber: 1) qual é o paradigma de interação mais eficiente para determinados públicos-alvo ao executar tarefas elementares de seleção; bem como 2) até que ponto as mesmas interfaces escolhidas para comparação poderão ter resultados díspares entre os vários grupos de participantes. Estas são as duas contribuições do presente trabalho.

Após apresentar um breve apanhado de trabalho relacionado, descrevemos a metodologia usada neste estudo e apresentamos e discutimos os respetivos resultados.

\section{TRABALHO RELACIONADO}

Podemos considerar como interação natural todas as interfaces input / output que possam incorporar gestos e linguagem corporal, fala, posição e aproximação de objetos, todo o espectro de output visual e auditivo, cheiro, tacto e perceção háptica [6]. Efetivamente, tanto as modalidades de input ativas como as passivas [16] têm sido implementadas em diversas áreas de atuação, como na educação, entretenimento, publicidade e outros, não só como interfaces independentes, mas também como um sistema multimodal. Com a evolução do estudo de interfaces multimodais, torna-se cada vez mais importante compreender os contextos e os comportamentos dos utilizadores [7], uma vez que se pode mostrar imperativo ter consciência do contexto mediante tarefas diárias, seja em dispositivos fixos como móveis [17].

As interfaces multitoque têm mostrado muito potencial principalmente no que concerne à sua facilidade de utilização, eficiência e natureza intuitiva, assim como a possibilidade de aumentar as taxas de produtividade relacionadas com o uso informativo [18]. Este tipo de interface tem sido contemplado em estudos respeitantes a diferentes contextos e faixas etárias (crianças [19]; idosos [20]), mas sempre como relatórios independentes e não como uma abordagem sistemática com a combinação de múltiplas modalidades de input.

Da mesma forma, também as interfaces tangíveis encorajam uma experiência interativa social e proporcionam ao utilizador a oportunidade de pensar sobre informações abstratas e virtuais de uma forma direta e palpável [21]. Assim, o utilizador consegue receber feedback imediato aos estímulos dados através de objetos físicos. Mais ainda, estudos indicam que o uso deste tipo de interface é muito apreciado principalmente pelas crianças [22]. Esta é uma ideia também apoiada neste estudo, como irá ser discutido na secção dos resultados.

Por outro lado, interfaces baseadas em gestos tridimensionais oferecem ao utilizador a liberdade e capacidade de manipular informações virtuais tirando partido da profundidade de um definido contexto espacial [23]. Tal como outras interfaces mencionadas anteriormente, também a gestual tem sido implementada num largo campo de diferentes áreas e contextos de atuação [24] e [25], bem como em faixas etárias mais alargadas [26]. Torna-se ainda relevante perceber até que ponto poderá ser complicada a adaptação a tais tipos de interface baseadas em gestos 3D, sem contacto físico com essa mesma interface [27].

Contudo, ainda se conhece muito pouco sobre como as diferentes interfaces podem influenciar o desempenho de uma pessoa no que diz respeito à sua idade. Tem sido desenvolvido trabalho nesta área, mas sempre como estudos independentes e dissociados, ou cobrindo apenas uma dupla de interfaces ou de idades [28]. De facto, têm-se realizado estudos quanto à resposta do utilizadores relativamente ao desempenho de determinadas tarefas com o rato versus outras interfaces: displays multitoque [29], [30]; interfaces tangíveis [31], [32]; ou mesmo entre si [33]. Além disso, algumas experiências revelaram resultados onde mais do que duas das mencionadas interfaces são comparadas em simultâneo [34], [35]; mas poucos estudos recentes têm sido desenvolvidos relativamente a uma avaliação sistemática das interfaces versus diferentes tarefas e perfis de utilizador. Ainda importante referir é o facto de que essas avaliações comparam diferentes interfaces, mas sempre com determinadas faixas etárias, e não entre estas.

Assim sendo, estudos publicados não forneceram ainda uma compreensão acerca da natureza ou da gravidade das dificuldades que diferentes utilizadores sentem quando utilizam distintos dispositivos de input. Mais ainda, não foram ainda realizadas comparações transversais entre diferentes faixas etárias num mesmo estudo. Este é, precisamente, o nosso ponto de ação nesta avaliação, que se situa no âmbito de um estudo sistemático ainda mais alargado sobre interfaces naturais que estamos a levar a cabo.

\section{METODOLOGIA}

De forma a analisar até que ponto utilizadores de grupos de idade distintos poderão ter a mesma experiência em termos de usabilidade, tentamos aqui identificar que modo de interação será o mais apropriado para a tarefa de seleção nos diferentes grupos, em termos de: eficiência (desempenho), eficácia (número de erros) e preferência do utilizador. Para tal, exploramos neste estudo a destreza dos participantes em quatro modos de input: o rato, toque, objetos tangíveis e gestos.

\section{A. Participantes:}

Participaram na experiência um total de 60 voluntários que desconheciam os propósitos do estudo, sendo estes divididos em 3 grupos de acordo com a sua idade:

- Crianças: grupo composto por 20 participantes com idades compreendidas entre os 9 e os 12 anos, frequentadores do quinto e sexto anos de escola primária.

- Jovens adultos: grupo composto por 20 participantes com idades compreendidas entre os 20 e os 30 anos, sendo estes estudantes universitários de cursos relacionados com informática.

- Adultos mais velhos: grupo composto por 20 participantes com idades compreendidas entre os 45 e os 60 anos, sendo estes trabalhadores ativos de um departamento de secretariado de uma escola.

Nesta fase, achamos importante perceber o nível de literacia digital dos participantes, bem como o seu à vontade com os diferentes modos de input. Esta informação foi 
recolhida no início dos testes e considera-se relevante para o processo de triagem do público-alvo a ser representado na experiência, uma vez que níveis diferentes de literacia poderiam influenciar os resultados. Assim sendo, averiguamos que todos os participantes tinham por hábito trabalhar com computadores diariamente e apresentavam o mesmo nível de literacia digital.

Além disso, é importante perceber quantos elementos do grupo já tinham tido experiência com os outros modos de interação para além do rato e qual a influência que essa experiência teve nos resultados individuais. Este pormenor será discutido na secção dos resultados. A Tabela I mostra a percentagem de participantes que já tinham tido experiência com as diferentes interfaces.

TABELA I. PERCENTAGEM DE PARTICIPANTES QUE JÁ TINHAM TIDO CONTACTO COM AS DIFERENTES INTERFACES.

\begin{tabular}{cccc}
\hline \hline INTERFACES & CRIANÇAS & $\begin{array}{c}\text { JOVENS } \\
\text { ADULTOS }\end{array}$ & $\begin{array}{c}\text { ADULTOS MAIS } \\
\text { VELHOS }\end{array}$ \\
\hline GRÁFICA & $100 \%$ & $100 \%$ & $100 \%$ \\
TOQUE & $95 \%$ & $100 \%$ & $65 \%$ \\
TANGÍVEL & $15 \%$ & $35 \%$ & $15 \%$ \\
GESTUAL & $40 \%$ & $60 \%$ & $15 \%$ \\
\hline
\end{tabular}

Como se pode constatar, a interação através do rato e toque são as mais familiares. Devido aos vários contextos de atividade, todos os participantes tinham experiência com o rato, seja pela necessidade de executar trabalhos e estudar, ou mesmo pela situação profissional. Quanto ao toque, 35\% dos adultos mais velhos confirmaram nunca ter experimentado um dispositivo de toque, apesar de já terem visto o processo noutras pessoas que possuíam tais displays. De uma forma geral, a interface com menor nível de experiência entre os três grupos é a tangível, talvez por não ser tão comum no meio considerado. Logo a seguir encontramos a interface gestual que aparenta ser familiar numa percentagem dos participantes, principalmente no grupo dos adultos. Percebemos ainda que esta situação se deve ao facto desta faixa etária se interessar por jogos de consola com o suporte desta modalidade. Também por este mesmo motivo os outros grupos tinham elementos na mesma circunstância, tendo estes experimentado interagir não só através de gestos, mas também de objetos tangíveis para cumprir os objetivos dos jogos de consola.

\section{B. Design experimental:}

A experiência abarcou um design within-subjects com uma análise de variância ANOVA para medidas repetidas. Cada um dos 60 participantes executou as tarefas solicitadas cinco vezes, o que resulta em 20 trials por sujeito, e um total de 1200 trials durante toda a experiência.

Para uma avaliação quantitativa, estabelecemos métricas de desempenho associadas às várias interfaces: consideramos a rapidez e a precisão do utilizador para completar a tarefa. Além disso, reunimos também informações qualitativas através de uma observação direta dos comportamentos dos participantes e ainda por meio de questionários realizados no final de cada teste, baseados em escalas de Likert e listas de ranking. Aqui tínhamos como objetivo perceber, para cada uma das modalidades de interação, a opinião dos participantes quanto a: facilidade de utilização, aprendizagem, efeito de fadiga, naturalidade de interação, nível de conforto / frustração, nível de concentração e, claro, a sua preferência.

\section{Cenário:}

Os testes foram realizados numa sala fechada com luz artificial e o setup, apresentado na Figura 1, consistia num ecrã de toque de 22', uma barra de sensor Kinect, uma webcam, oito peças tangíveis marcadas com fiduciais e um rato de computador.

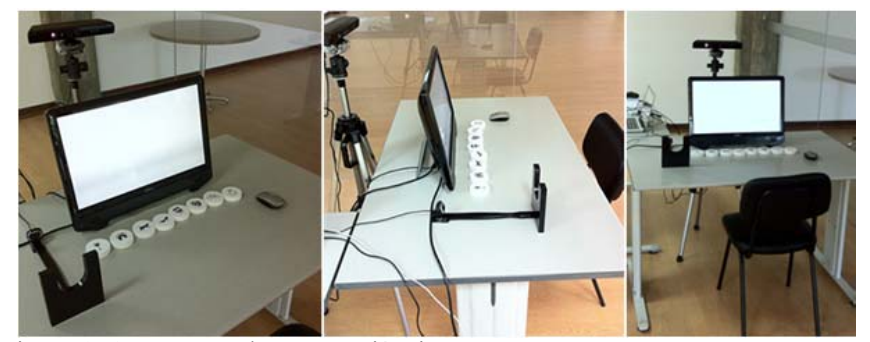

Figura 1. Aparato usado na experiência.

O sensor Kinect estava montado num tripé atrás do ecrã, cerca de $25 \mathrm{~cm}$ acima deste, e virado de encontro ao utilizador de forma a conseguir detetar a posição da sua mão e o seu movimento. Quanto às peças tangíveis, estas estavam colocadas em cima da mesa posicionadas em frente ao ecrã, organizadas pela mesma ordem e com um espaçamento uniforme.

Além disso, o software usado na experiência foi criado especificamente para este fim. As tarefas foram desenvolvidas em Python, recorrendo à framework Kivy, o rastreio dos fiduciais foi gerado pela framework opensource reacTIVision [36] e, por fim, o rastreio dos gestos e movimentos das mãos dos participantes era feito através da aplicação TuioKinect [37]. Ambas as implementações enviavam os dados por TUIO para a aplicação principal.

\section{Procedimento}

$\mathrm{O}$ primeiro passo da experiência concentrou-se em explicar aos participantes uma visão geral das tarefas a desempenhar, assim como quais as modalidades de input disponíveis para a seleção. Ainda nesta etapa era lançado um questionário individual para averiguar o nível de experiência prévia com as diferentes interfaces.

$\mathrm{O}$ investigador estava continuamente presente durante os testes de forma a: 1) explicar cada uma das tarefas e, cada vez que era introduzida uma nova possibilidade de interação, como esta funcionava e como a usar; 2) retirar elações sobre o comportamento dos participantes e suas reações durante a interação.

A experiência foi dividida em quatro fases distintas, cada uma fazendo uso de uma modalidade de interação diferente para desempenhar a tarefa de seleção:

- Tarefa 1: Recorrer ao rato para a seleção.

- Tarefa 2: Recorrer ao toque para a seleção.

- Tarefa 3: Recorrer a objetos tangíveis para a seleção.

- Tarefa 4: Recorrer a gestos (movimentação) da mão para a seleção.

A finalidade de cada tarefa era a seleção do botão correto correspondente ao solicitado pelo investigador de entre oito 
exibidos no ecrã. Estes botões estavam organizados em duas colunas com quatro botões cada, e eram posicionados na grelha aleatoriamente.

Ao iniciar a aplicação, o primeiro ecrã correspondia a um contador iniciado no número $3 \mathrm{e}$ em contagem decrescente até 1. No término do contador era exibida no ecrã a grelha de botões, e apenas então começava a ser contabilizado o tempo de interação. $\mathrm{O}$ nome do botão pretendido era dito aos participantes no preciso momento de término da contagem decrescente, incentivando uma reação mais rápida de seleção do botão correto. Era ainda solicitado aos sujeitos que desempenhassem a tarefa o mais rapidamente possível e com o menor número de erros.

Após a seleção do botão correto era exibida ao utilizador uma mensagem de "tarefa concluída com sucesso" e, por conseguinte, este passaria para o trial seguinte. Pelo contrário, se o botão selecionado não fosse o esperado o ecrã exibiria uma mensagem de "botão incorreto", bem como um aviso de que a tarefa iria reiniciar dentro de cinco segundos. Esta nova oportunidade de seleção era dada a utilizador por cada vez que errasse o botão, e todos os trials de erro eram contabilizados automaticamente pela aplicação.

A primeira e segunda tarefas consistiam na utilização do rato e toque, respetivamente, como modalidade de input. Já a terceira tarefa abarcava a interação através de objetos tangíveis, sendo necessário para a seleção do botão correto a escolha de uma das oito peças físicas disponíveis rotuladas com os ícones correspondentes aos nomes dos botões (Figura 2). Era pedido aos participantes que levantassem a peça pretendida e a inserissem num mecanismo com uma webcam preparado especificamente para o estudo. Aqui, o sistema iria reconhecer o ID do fiducial gravado no verso da peça.
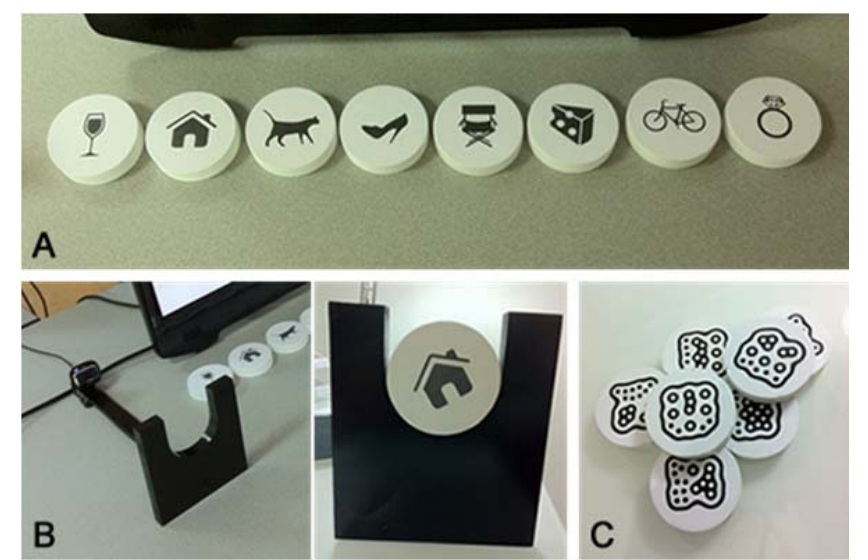

Figura 2. (a) Ordem das peças tangíveis usadas na experiência; (b) Aparelho com a webcam adaptada para reconhecimento dos fiduciais; (c) Fiduciais rotulados no verso das peças.

A quarta e última tarefa consistia em selecionar os botões recorrendo a movimentos com a mão (gestos 3D). Era pedido ao utilizador que levantasse o seu braço e o mantivesse erguido dentro de uma área de segurança entre ele e o ecrã. $\mathrm{O}$ sensor Kinect iria, aqui, detectar a posição da sua mão e eram enviadas as suas coordenadas para a aplicação que, nesse sentido, exibia no ecrã uma imagem de um alvo de forma a mostrar que o sistema estava de facto a reconhecer o gesto de apontar. A finalidade era selecionar o botão ao mover o alvo com o gesto da mão para cima do botão pretendido, apontando e esperando nessa mesma posição durante 0,8 segundos. $\mathrm{O}$ sistema iria assim reconhecer a intenção do utilizador. Esta estratégia de "point and wait" para a seleção com gestos foi elegida com base no estudo de [38]. A imagem do alvo no ecrã proporcionava feedback visual ao participante para que este se apercebesse do progresso da seleção: quando o sujeito mantinha a sua mão imóvel numa determinada posição, um círculo verde começaria a crescer no interior do alvo para indicar a seleção. Este referido tempo de espera para a seleção ser feita foi retirado da variável de tempo total por motivos de coerência na avaliação.

Não foi dado aos participantes um limite de tempo para completar a tarefa, mas era-lhes transmitida a importância de a terminar o mais rapidamente possível, evitando o maior número de erros. Além disso, uma vez que se poderia tornar num teste bastante desgastante para determinados públicosalvo (sobretudo em crianças), os sujeitos poderiam descansar a qualquer momento entre as tarefas.

Um dos objetivos do estudo era também perceber até que ponto os participantes com alguma experiência com outras interfaces além do rato, e aqueles que nunca tinham tido contacto com essas modalidades de input, teriam tempos de desempenho e reações diferentes, bem como qual o tempo necessário de adaptação à nova modalidade. Também por esse motivo, e para perceber se o desempenho iria melhorar com a prática, ou não, todas as tarefas eram repetidas cinco vezes.

Optamos ainda por manter a ordem das tarefas inalterável por motivos de perceber até que ponto as interfaces poderiam ser intuitivas e de fácil utilização, mesmo sem experiência prévia com a modalidade. Como tal, nesta situação o participante poderia compreender bem a estrutura dos testes e por exemplo, na última fase apenas se iria concentrar na seleção do botão correto através dos gestos, não perdendo tempo em nenhuma parte do processamento das instruções para completar a tarefa. Como iremos perceber ainda na secção dos resultados, a questão da interface gestual ser a última a ser desempenhada não aparenta ter influenciado uma diminuição nos tempos médios de seleção.

Após a conclusão dos testes, era solicitado aos participantes o preenchimento de um outro questionário relacionado com a sua opinião acerca das várias modalidades de input. A nossa intenção era recolher a perspectiva dos participantes quanto à sua própria eficiência e desempenho.

\section{RESULTADOS}

Nesta secção, apresentamos os resultados obtidos com este estudo. Não apreciamos nenhum outlier, pelo que os dados recolhidos estavam normalmente distribuídos em cada um dos grupos. Acreditamos que poderemos conseguir lançar ideias sobre como diferentes grupos de utilizadores em termos de idade apreendem as várias modalidades de interação, e qual será a mais eficiente para uma tarefa de seleção mediante cada perfil. 
TABELA II. RESULTADOS DOS TEMPOS DE REALIZAÇÃO DA TAREFA (POR GRUPO).

\begin{tabular}{|c|c|c|c|c|c|}
\hline$\overline{\text { GRUPOS }}$ & $\begin{array}{l}\text { MODALIDADE DE } \\
\text { INPUT }\end{array}$ & TEMPO MÉDIO & "TEMPO MÁXIMO & TEMPO MÍNIMO & " DESVIO PADRÃO \\
\hline \multirow{4}{*}{ CRIANÇAS } & RATO & 2,69 & 4,67 & 0,79 & 0,82 \\
\hline & TOQUE & 2,27 & 3,77 & 0,98 & 0,66 \\
\hline & TANGÍVEL & 4,16 & 7,75 & 1,98 & 1,26 \\
\hline & GESTOS & 9,12 & 16,67 & 2,72 & 2,72 \\
\hline \multirow{4}{*}{$\begin{array}{c}\text { JOVENS } \\
\text { ADULTOS }\end{array}$} & RATO & 2,12 & 3,45 & 0,78 & 0,65 \\
\hline & TOQUE & 2,10 & 2,98 & 1,13 & 0,46 \\
\hline & TANGÍVEL & 3,61 & 4,74 & 2,32 & 0,65 \\
\hline & Gestos & 8,00 & 11,75 & 2,49 & 0,65 \\
\hline \multirow{4}{*}{$\begin{array}{c}\text { Adultos MAIS } \\
\text { VelHos }\end{array}$} & RATO & 3,25 & 5,74 & 0,90 & 1,00 \\
\hline & TOQUE & 2,08 & 3,73 & 1,11 & 0,57 \\
\hline & TANGÍVEL & 3,84 & 5,94 & 2,72 & 1,01 \\
\hline & Gestos & 9,63 & 12,14 & 2,72 & 3,21 \\
\hline
\end{tabular}

\section{A. Desempenho de cada grupo}

Relativamente às diferentes interfaces, analisamos o tempo de realização da tarefa em cada grupo de participantes e ainda entre os vários grupos. Este tempo de realização foi medido a partir do momento que eram exibidos os botões no ecrã, até que um deles era selecionado com sucesso e aparecia a mensagem de conclusão da tarefa. A Tabela II mostra a média (em segundos) dos tempos de cada grupo nas diversas interfaces, bem como o tempo máximo e mínimo registado, e o desvio padrão dos valores.

Em relação ao grupo das crianças, a modalidade de input com um tempo médio mais rápido foi o toque $(2,27$ seg.), seguido pelo rato (2,69 seg.). As duas interfaces com os resultados mais lentos foram a tangível e a gestual com valores de 4,16 seg. e 9,12 seg., respetivamente. Uma análise de variância ANOVA para medidas repetidas com uma correção de Greenhouse-Geisser determinou uma diferença significativa entre os tempos de realização da tarefa em cada uma das modalidades de interação $(\mathrm{f}(1.108,21.044)=19.904$, $\mathrm{p}<.000)$. Testes comparativos emparelhados post-hoc com uma correção Bonferroni mostrou que, com a exceção do rato $\mathrm{e}$ do toque $(\mathrm{p}=.353)$, os tempos médios foram significativamente diferentes: rato e as peças tangíveis $(\mathrm{p}=$ $0.001)$; rato e gestos $(\mathrm{p}=.000)$; toque versus peças tangíveis / gestos $(\mathrm{p}=.000)$; tangíveis e gestos $(\mathrm{p}=.000)$.

Quanto aos resultados do grupo dos jovens adultos, o toque foi igualmente a modalidade de interação que registou a média de tempo de realização mais baixa em todas as tarefas $(2,10$ seg.), seguido com uma diferença mínima de valores pelo rato (2,12 seg.). Novamente neste grupo, as interfaces que registaram tempos médios mais altos foram: a tangível com 3,16 seg., e a gestual com 8,00 seg. De facto, este grupo mostrou uma diferença insignificante entre o rato e o toque (,02 seg.), o que poderá indicar que, neste particular grupo de idades, será indiferente o uso do rato ou toque para desempenhar uma tarefa de seleção. Também aqui procedemos a uma análise de variância ANOVA para medidas repetidas e, uma vez que não foi assumida a esfericidade de Mauchly's, aplicamos a correção de Greenhouse-Geisser (f $(1.010,19.190)=12.520, \mathrm{p}<.002)$. Em relação ao toque $\mathrm{e}$ rato, este grupo mostrou uma diferença mínima quanto à média de tempo de realização, que se provou não ser estatisticamente significante por meio de testes comparativos emparelhados usando a correção de Bonferroni $(\mathrm{p}=1.000)$. Esta diferença mostrou-se ainda menor do que o grupo anterior. Mais ainda, a diferença entre os gestos e as peças tangíveis não se mostrou igualmente como estatisticamente significativa $(\mathrm{p}=.077)$, ao contrário das outras combinações: rato e gestos $(\mathrm{p}=.008)$; toque e gestos $(\mathrm{p}=.003)$; e as peças tangíveis versus o rato ou o toque $(\mathrm{p}=.000)$.

Já o desempenho do grupo dos adultos mais velhos também alcançou uma diferença estatisticamente significativa entre as várias interfaces $(\mathrm{f}(1.137,21.606)=32.007, \mathrm{p}<.000)$, tal como determinado por uma análise ANOVA para medidas repetidas com uma correção de Greenhouse-Geisser. De acordo com testes comparativos emparelhados post-hoc com uma correção Bonferroni, os tempos médios de realização das tarefas foram reportados como estatisticamente diferentes, à exceção da combinação de peças tangíveis e o rato $(\mathrm{p}=.373)$. Assim sendo, a conjugação do rato e gestos apresentou diferenças significativas $(\mathrm{p}=.000)$, tal como o toque versus peças tangíveis / gestos $(\mathrm{p}=.000)$, e tangíveis e gestos $(\mathrm{p}=$ .001). Neste caso, contrariamente aos outros grupos, a diferença entre o rato e toque como modalidades de input mostrou-se como estatisticamente significativa $(\mathrm{p}=.003)$. Efetivamente, este grupo registou um padrão de desempenho diferente em comparação com os outros dois grupos: o rato e as peças tangíveis não assinalaram uma diferença significativa, com um tempo médio de apenas 0,59 seg. de intervalo entre si. Da mesma forma, também muito díspar em relação aos outros grupos se apresentou a combinação do rato e toque, que aqui apresentaram resultados estatisticamente diferentes com um intervalo entre eles de $1,17 \mathrm{seg}$. de tempo médio. Este resultados impeliram-nos no sentido de existir possivelmente um contraste entre grupos no que concerne à modalidade de interação e o perfil etário dos utilizadores. Por fim, a interface com o tempo médio mais baixo foi a táctil (2,08 seg.), seguida pelo rato (3,25 seg.), a tangível (3,84 seg.) e a gestual $(9,63$ seg.).

\section{B. Desempenho global entre grupos}

Além de perceber o desempenho de cada um dos grupos nas várias interfaces, também comparamos o desempenho 
entre os três grupos. A Figura 3 mostra visualmente o tempo médio gasto para completar a tarefa pelos três grupos, em cada interface.

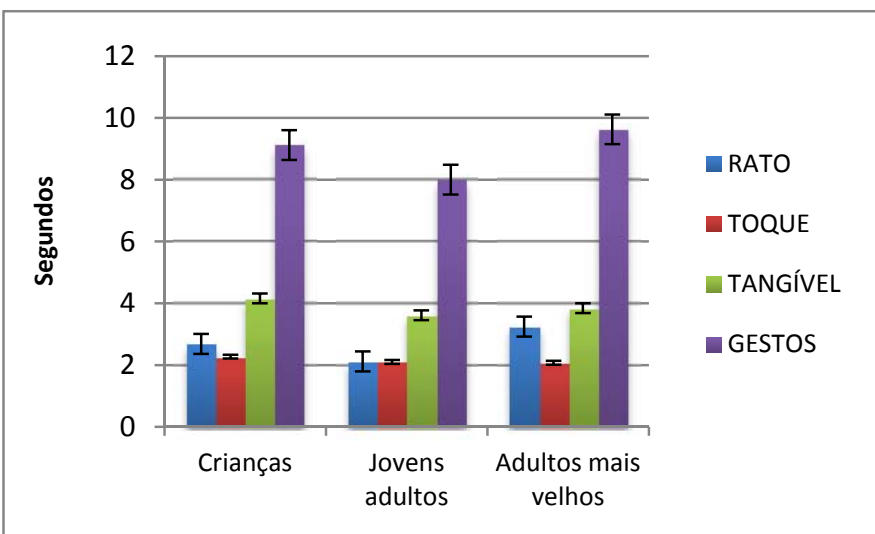

Figura 3. Médias dos tempos de realização de cada grupo (em segundos).

Efetivamente, após análise detetámos que não existe uma diferença estatisticamente significativa entre os grupos em cada modalidade de input, com exceção do rato. Este registou uma diferença significativa entre os grupos dos jovens adultos e adultos mais velhos $(\mathrm{p}=.002)$. Curiosamente, o único dispositivo de input com que todos os participantes tinham habituação e utilização diária - o rato - foi precisamente aquele que mostrou resultados mais díspares entre os grupos.

De uma maneira geral, o grupo dos jovens adultos foi aquele que apresentou tempos médios de realização mais baixos na maioria das modalidades de interação. Além disso, os tempos médios de seleção das várias modalidades seguiram um padrão nos três grupos: 1) a interface gestual mostrou-se como menos eficiente do que as restantes, apresentando grandes discrepâncias em comparação com as quatro modalidades de input em termos de tempos médios; e 2) o toque foi o mais rápido e consistente entre os grupos, com pequenas variações em tempo médio. Mais ainda, para a tarefa elementar de seleção, o toque apresenta indícios de ser mais eficiente (com os tempos de realização mais baixos) em todos os grupos, mesmo considerando que a experiência com esta modalidade não era global (como concluído nos inquéritos iniciais). Poderemos, assim, retirar elações que o tipo / perfil do utilizador poderá não influenciar o desempenho na realização de uma tarefa, mas sim o tipo de interface utilizada; ou seja, de acordo com um determinado tipo de tarefa, o recurso a diferentes interfaces poderá influenciar o desempenho do utilizador e a sua preferência. Mesmo comparando os grupos diretamente, chegamos à conclusão que nem todas as interfaces produziram os mesmos resultados.

\section{Número de erros}

Consideramos um erro quando o botão selecionado pelo participante não era, efetivamente, o correto. A única modalidade de interação que registou faltas durante a tarefa, sendo assim, foi a gestual, com um total de 2 erros do grupo das crianças, 8 erros por parte dos jovens adultos, e 14 dos adultos mais velhos. O grupo dos adultos mais velhos registou, assim, a maior taxa de erros de entre os três grupos, mas também a média mais alta de tempos de seleção.

\section{Preferências dos participantes}

No final da experiência, era solicitado aos participantes que preenchessem um inquérito em relação à sua perspectiva sobre o seu próprio desempenho e suas preferências.

Quando confrontados sobre qual a interface que mais gostaram quanto à facilidade de utilização e caráter intuitivo, a maioria escolheu a tátil. Mesmo para os participantes que nunca tinham utilizado o toque como modalidade de interação, este foi o mais popular, além de que todos os participantes referiram que era fácil de perceber e prático para uma utilização diária. Já quanto ao conforto de interação, todos os participantes referiram a interface gestual como a menos confortável e mais desafiante de todas. Ainda sobre a interface gestual, os grupos dos jovens adultos e adultos mais velhos assentiram que esta era a mais exigente em termos de concentração necessária para completar a tarefa.

Em termos de preferência de utilizador, as crianças escolheram o toque e as peças tangíveis como as duas modalidades favoritas, e os restantes dois grupos escolheram o toque como predileto. De facto, a maioria das crianças escolheu o toque como favorito, com $60 \%$ das respostas, seguido logo pela interface tangível (com 25\%), indo este resultado de encontro ao estudo de [22]. No final das preferências posicionaram-se os gestos e o rato, com $40 \%$ e $30 \%$ de respostas, respetivamente. Da mesma forma, também a maioria dos jovens adultos elegeu o toque como favorito com $65 \%$ das respostas, e $60 \%$ deles apontaram a interface gestual como a menos preferida. $\mathrm{O}$ grupo dos adultos mais velhos optou igualmente pelo toque (75\%) como modalidade favorita, deixando para trás o toque e os gestos $(10 \%$ de respostas cada), e apenas $5 \%$ para a interface tangível.

\section{E. Análise qualitativa}

Consideramos importante divulgar um conjunto de comportamentos resultantes dos participantes e recolhidos por meio de uma observação participante.

Numa primeira etapa, durante a utilização do rato, todos os participantes mostraram um padrão na sua abordagem à tarefa: procuravam no ecrã o botão correto e depois concluíam a tarefa clicando neste. Contudo, notámos um comportamento variável no grupo das crianças e dos adultos mais velhos: estes dedicavam inicialmente o seu tempo a procurar a posição do cursor no ecrã, e apenas depois de o encontrar tentavam detetar o botão correto.

Aquando da interação por toque e através das peças tangíveis, os grupos demonstraram um comportamento semelhante entre si, não demonstrando esforço atípico em completar as tarefas.

A tarefa relacionada com a interface gestual provou ser a mais desafiante, especialmente aquando da necessidade de selecionar botões posicionados nas extremidades superiores do ecrã (cantos superiores esquerdo e direito). O comportamento mais vulgar nesta situação era o esticar do braço de encontro ao ecrã numa direção horizontal, com o intuito de selecionar o botão e saindo, assim, da área de deteção, em vez de o 
levantar com um movimento ascendente, numa direção vertical. Esta circunstância levava não raras vezes o participante a um nível de frustração mais elevado, acabando por afetar os restantes trials da tarefa até a concluir com sucesso.

Por outro lado, não aparentou existir nenhuma diferença de comportamento no que concerne aos utilizadores destros ou canhotos. Ambos demonstraram um desempenho nas tarefas com semelhante conforto.

\section{DISCUSSÃO}

Quanto aos resultados relativos à utilização do rato como dispositivo de input, e uma vez que esta modalidade se apresenta como aquela em que todos os participantes tinham prática, a questão dos tempos médios terem sido muito inferiores no grupo dos adultos mais velhos dá-nos a entender que esta modalidade de interação poderá sofrer ao longo dos anos um detrimento de desempenho por parte dos utilizadores em vez de, pelo contrário, aumentar com a experiência. Também relativamente à interface gestual, poderemos inferir que talvez a destreza deste grupo de participantes possa diminuir ao longo dos anos: principalmente com a interação gestual, notámos que os reflexos motores poderão ter sido influenciados pela idade, uma vez que se tornava mais lenta a movimentação da mão para evitar localizações específicas que despoletavam a animação do alvo para selecionar o botão sem intenção. Não iremos, contudo, aprofundar este tópico neste estudo.

De uma maneira geral, apercebemo-nos que, com a repetição dos trials em cada tarefa, a recorrência da ação a desempenhar não provocava uma diminuição dos tempos médios de seleção. Efetivamente, não podemos extrapolar uma conclusão neste sentido, uma vez que a repetição constante de uma tarefa poderia significar um aumento de experiência e, logo, melhores resultados. Contudo, não era nossa prioridade perceber até que ponto os tempos médios diminuíam com a experiência, mas sim perceber os tempos de adaptação do utilizador a uma determinada interface, e perceber até que ponto esta se pode considerar intuitiva e sem necessidade de treino prévio (por essa razão optamos por não fornecer um tempo de treino entre as tarefas). Tal facto comprova-se com o desempenho dos participantes com o rato onde, mesmo com um nível de experiência similar entre eles, apresentou resultados significativamente diferentes.

Podemos aqui ter indícios, assim, de que a interface tátil seria a mais intuitiva e de fácil adaptação, uma vez que teve melhores resultados nos vários grupos, mesmo sem todos os participantes apresentarem o mesmo contacto com esta. Contudo, em termos de preferência de utilizador pode ser importante analisar melhor as respostas e escolhas de interface favorita, uma vez que pode representar uma luz sobre que interface seria melhor acolhida em determinadas faixas etárias.

\section{CONCLUSÕES}

Esta investigação exploratória tem a intenção de compreender qual a modalidade de interação mais eficiente para um determinado público-alvo numa tarefa de seleção.
Apesar de se apresentar ainda como uma primeira fase de um estudo exploratório mais exaustivo a todas as tarefas elementares, e não apenas à de seleção, de acordo com os resultados aqui expostos parecem existir indícios que a criação de perfis de utilizador distintos mediante a interação com diferentes modalidades de input pode ser importante. Mais ainda, os resultados acentuaram a importância de uma investigação mais aprofundada sobre a relação entre a idade dos utilizadores e as diferentes modalidades de input.

Ainda que os resultados tenham indiciado serem importantes para perceber que rumo tomar quanto à escolha da interfaces mediante o perfil do utilizador, este estudo não irá cingir-se apenas a uma avaliação de usabilidade. Deverá, sim, tomar também em consideração uma avaliação de aquisição de alvos e desempenho com dispositivos apontadores, com o recurso à Fitts' Law [39], que será considerado o próximo passo na investigação.

Os resultados aqui discutidos devem ser entendidos como uma chamada de atenção para novos estudos a respeito das diversas tarefas a desempenhar, bem como uma maior exploração da influência da idade na interação e no desempenho dos utilizadores, comparando as diversas interfaces e tarefas com vários grupos de utilizadores, e observando as diferenças de interação que surgem entre si.

\section{AGRADECIMENTOS}

Gostaríamos de agradecer todo o apoio e a contribuição da Universidade de Trás-os-Montes e Alto Douro (UTAD) e às escolas que participaram neste estudo: Colégio Moderno de São José e Escola Secundária Morgado de Mateus, ambas em Vila Real, Portugal.

Mais ainda, Diana Carvalho tem uma Bolsa de Doutoramento concedida pela FCT - Fundação para a Ciência e a Tecnologia (SFRH/BD/81541/2011).

\section{REFERÊNCIAS}

[1] J. Nielsen, "Noncommand user interfaces," Commun. ACM, vol. 36, no. 4, pp. 83-99, Apr. 1993.

[2] C. Stephanidis, "User Interfaces for All: New Perspectives into HumanComputer Interaction," in User Interfaces for All - Concepts, Methods, and Tools, C. Stephanidis, Ed. Mahwah, NJ: Lawrence Erlbaum Associates, 2001, p. 760.

[3] A. van Dam, "Post-WIMP user interfaces," Commun. ACM, vol. 40, no. 2, pp. 63-67, 1997.

[4] J. Blake, "The natural user interface revolution," in Natural User Interfaces in .NET, Manning Publications Co, 2012, pp. 1-43.

[5] R. E. Mayer and R. Moreno, "Nine Ways to Reduce Cognitive Load in Multimedia Learning," Educ. Psychol., vol. 38, no. 1, pp. 43-52, Mar. 2003.

[6] J. Jain, A. Lund, and D. Wixon, "The future of natural user interfaces," in Proceedings of the 2011 annual conference extended abstracts on Human factors in computing systems - CHI EA '11, 2011, p. 211.

[7] S. Oviatt and P. Cohen, "Perceptual user interfaces: multimodal interfaces that process what comes naturally," Commun. ACM, vol. 43, no. 3, pp. 45-53, Mar. 2000.

[8] S. Oviatt, "Mulitmodal Interactive Maps: Designing for Human Performance," Human-Computer Interact., vol. 12, no. 1, pp. 93-129, Mar. 1997.

[9] A. M. Piper, N. Weibel, and J. D. Hollan, "Write-N-Speak," $A C M$ Trans. Access. Comput., vol. 4, no. 1, pp. 1-20, Nov. 2011.

[10] J. Herold and T. F. Stahovich, "Using speech to identify gesture pen strokes in collaborative, multimodal device descriptions," Artif. Intell. Eng. Des. Anal. Manuf., vol. 25, no. 03, pp. 237-254, Jul. 2011. 
[11] M. Miki, N. Kitaoka, C. Miyajima, T. Nishino, and K. Takeda, "Improvement of multimodal gesture and speech recognition performance using time intervals between gestures and accompanying speech," EURASIP J. Audio, Speech, Music Process., vol. 2014, no. 1, p. 2, 2014.

[12] J. Liu and M. Kavakli, "A survey of speech-hand gesture recognition for the development of multimodal interfaces in computer games," in 2010 IEEE International Conference on Multimedia and Expo, 2010, pp. 1564-1569.

[13] A. Lucchi, P. Jermann, G. Zufferey, and P. Dillenbourg, "An empirical evaluation of touch and tangible interfaces for tabletop displays," in Proceedings of the fourth international conference on Tangible, embedded, and embodied interaction - TEI '10, 2010, p. 177.

[14] I. C. Olson, Z. Atrash Leong, U. Wilensky, and M. S. Horn, "It's just a toolbar!," in Proceedings of the fifth international conference on Tangible, embedded, and embodied interaction - TEI '11, 2011, p. 29.

[15] D. A. Bowman, E. Kruijff, J. J. LaViola, and I. Poupyrev, "An Introduction to 3-D User Interface Design," Presence Teleoperators Virtual Environ., vol. 10, no. 1, pp. 96-108, Feb. 2001.

[16] S. L. Oviatt, "Multimodal Interfaces," in The Human-Computer Interaction Handbook: Fundamentals, Evolving Technologies and Emerging Applications, Second Edition, 2nd ed., A. Sears and J. A. Jacko, Eds. CRC Press, 2007, pp. 413-432.

[17] T. Siu and V. Herskovic, "Mobile augmented reality and contextawareness for firefighters," IEEE Lat. Am. Trans., vol. 12, no. 1, pp. 42-47, Jan. 2014

[18] K. Kin, M. Agrawala, and T. DeRose, "Determining the benefits of direct-touch, bimanual, and multifinger input on a multitouch workstation," pp. 119-124, May 2009.

[19] Xiaohua Yu, Mian Zhang, Yaofeng Xue, and Zhiting Zhu, "An exploration of developing multi-touch virtual learning tools for young children," in 2010 2nd International Conference on Education Technology and Computer, 2010, vol. 3, pp. V3-4-V3-7.

[20] D. Carvalho, M. Bessa, L. Oliveira, C. Guedes, E. Peres, and L. Magalhães, "New Interaction Paradigms to Fight the Digital Divide: A Pilot Case Study Regarding Multi-Touch Technology," Procedia Comput. Sci., vol. 14, pp. 128-137, Jan. 2012.

[21] M. J. Kim and M. Lou Maher, "Collaborative Design in a Tabletop System employing Tangible User Interfaces," in 2007 11th International Conference on Computer Supported Cooperative Work in Design, 2007, pp. 192-197.

[22] C. Sylla, P. Branco, C. Coutinho, and M. E. Coquet, "Storytelling through drawings," in Proceedings of the 27th international conference extended abstracts on Human factors in computing systems - CHI EA '09, 2009 , p. 3461.

[23] O. Hilliges, S. Izadi, A. D. Wilson, S. Hodges, A. Garcia-Mendoza, and A. Butz, "Interactions in the air," in Proceedings of the 22nd annual ACM symposium on User interface software and technology - UIST '09, 2009, p. 139.

[24] F. Tecchia, L. Alem, and W. Huang, "3D helping hands," in Proceedings of the 11th ACM SIGGRAPH International Conference on Virtual-Reality Continuum and its Applications in Industry - VRCAI '12, 2012, p. 323

[25] R. Walter, G. Bailly, and J. Müller, "StrikeAPose," in Proceedings of the SIGCHI Conference on Human Factors in Computing Systems CHI'13, 2013, p. 841.

[26] K. Gerling, I. Livingston, L. Nacke, and R. Mandryk, "Full-body motion-based game interaction for older adults," in Proceedings of the 2012 ACM annual conference on Human Factors in Computing Systems - CHI '12, 2012, p. 1873.

[27] O. Erazo and J. Pino, "Estimating the Difficulty of Touchless Hand Gestures," IEEE Lat. Am. Trans., vol. 12, no. 1, pp. 17-22, Jan. 2014.

[28] L. Findlater, J. E. Froehlich, K. Fattal, J. O. Wobbrock, and T. Dastyar, "Age-related differences in performance with touchscreens compared to traditional mouse input," in Proceedings of the SIGCHI Conference on Human Factors in Computing Systems - CHI '13, 2013, p. 343.

[29] E. Beheshti, A. Van Devender, and M. Horn, "Touch, click, navigate," in Proceedings of the 2012 ACM international conference on Interactive tabletops and surfaces - ITS '12, 2012, p. 205.

[30] C. Forlines, D. Wigdor, C. Shen, and R. Balakrishnan, "Direct-touch vs. mouse input for tabletop displays," in Proceedings of the SIGCHI conference on Human factors in computing systems - CHI '07, 2007, p. 647
[31] A. N. Antle, M. Droumeva, and D. Ha, "Hands on what?," in Proceedings of the 8th International Conference on Interaction Design and Children - IDC '09, 2009, p. 80.

[32] L. Xie, A. N. Antle, and N. Motamedi, "Are tangibles more fun?: comparing children's enjoyment and engagement using physical, graphical and tangible user interfaces," in Proceedings of the 2nd international conference on Tangible and embedded interaction - TEI '08, 2008, p. 191.

[33] P. Tuddenham, D. Kirk, and S. Izadi, "Graspables revisited," in Proceedings of the 28th international conference on Human factors in computing systems - CHI '10, 2010, p. 2223.

[34] T. J. Donahue, G. M. Poor, M. E. Mott, L. M. Leventhal, G. Zimmerman, and D. Klopfer, "On interface closeness and problem solving," in Proceedings of the 7th International Conference on Tangible, Embedded and Embodied Interaction - TEI '13, 2013, p. 139.

[35] A. Esteves, E. van den Hoven, and I. Oakley, "Physical games or digital games?: comparing support for mental projection in tangible and virtual representations of a problem-solving task," in Proceedings of the 7th International Conference on Tangible, Embedded and Embodied Interaction - TEI '13, 2013, p. 167.

[36] "reacTIVision, a toolkit for tangible multi-touch surfaces." [Online] Available: http://reactivision.sourceforge.net/. [Accessed: 18-Dec2014].

[37] "TuioKinect - A simple TUIO hand tracker for Kinect - Google Project Hosting." [Online]. Available: https://code.google.com/p/tuiokinect/. [Accessed: 18-Dec-2014].

[38] E. Schapira and R. Sharma, "Experimental evaluation of vision and speech based multimodal interfaces," in Proceedings of the 2001 workshop on Percetive user interfaces - PUI '01, 2001, p. 1.

[39] P. M. Fitts, "The information capacity of the human motor system in controlling the amplitude of movement.," J. Exp. Psychol., vol. 47, no. 6, pp. 381-391, 1954.

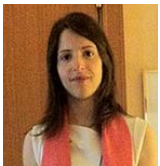

Diana Carvalho é estudante de Doutoramento em Informática na Universidade de Trás-os-Montes e Alto Douro (UTAD), em Vila Real, Portugal, e investigadora júnior no INESC Tec, laboratório associado do INESC Porto, no Porto, Portugal. Obteve o título de mestre em Multimédia pela Faculdade de Engenharia da Universidade do Porto (FEUP), Portugal. A sua área de interesse é em Interação Pessoa-Computador, em particular em Interfaces Naturais.

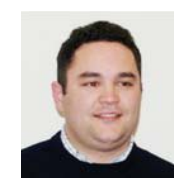

Maximino Bessa obteve o título de Doutor em Ciências Exactas, Naturais e Tecnológicas - Ciências da Engenharia pela Universidade de Trás-os-Montes e Alto Douro (UTAD), em Vila Real, Portugal. Atualmente é professor auxiliar na UTAD e investigador no INESC Tec, laboratório associado do INESC Porto, no Porto, Portugal. As suas principais áreas de pesquisa concentram-se no âmbito do Processamento de Imagem.

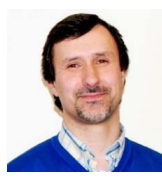

Luís Magalhães é atualmente professor assistente do Departamento de Sistemas de Informaão (DSI), na Universidade do Minho (UMinho), em Braga, Portugal, e investigador no INESC Tec, laboratório associado do INESC Porto, no Porto, Portugal. Foi ainda professor na Universidade de Trás-os-Montes e Alto Douro (UTAD) até ao ano letivo de 2013/2014. As suas principais áreas de pesquisa concentramse no âmbito de Computer Graphics.

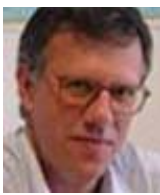

Eurico Carrapatoso é atualmente professor auxiliar do Departamento de Engenharia Eletrotécnica e de Computadores, na Faculdade de Engenharia da Universidade do Porto (FEUP), no Porto, Portugal. E ainda membro integrado do Instituto de Engenharia de Sistemas e Computadores do Porto, e investigador no INESC Tec, laboratório associado do INESC Porto, no Porto, Portugal. 